\title{
Optical and IR emission of a sample of IRAS galaxies
}

\author{
S. Lípari, ${ }^{1}{ }^{\star}$ Ch. Bonatto ${ }^{2}$ and M. G. Pastoriza ${ }^{2}$ \\ ${ }^{1}$ Observatório de Córdoba, Laprida 854, 5000 Córdoba, Argentina \\ ${ }^{2}$ Departamento de Astronomia, IF-UFRGS, Campus do Vale, CP 15051, Porto Alegre-RS, Brasil
}

Accepted 1991 June 5. Received 1991 June 5; in original form 1990 August 7

\begin{abstract}
SUMMARY
We report on observations of 12 southern warm IRAS galaxies. Using absolute fluxes of lines in the optical region in connection with diagnostic diagrams, we have classified these galaxies in terms of the principal excitation mechanisms and have found that all the galaxies are Seyferts. Far-infrared colours also are used to analyse these objects. Good correlations between the luminosity at 25 and $60 \mu \mathrm{m}$ and the $\mathrm{H} \alpha$ luminosity are found. The far-infrared luminosity of Seyfert galaxies seems to depend more strongly on the number of ionizing photons than on the amount of dust.
\end{abstract}

\section{INTRODUCTION}

Seyfert galaxies are known to have strong IR emission (Miley, Neugebauer \& Soifer 1985) and a method has been developed for selecting Seyfert candidates from their emission at 25 and $60 \mu \mathrm{m}$ (de Grijp et al. 1985; de Grijp, Miley \& Lub 1987). Osterbrock \& de Robertis (1985, hereafter OR85) have also confirmed, from a sample of 30 galaxies, that a large number of IRAS warm galaxies have active galactic nuclei (AGN) and that many of them are heavily reddened. Leech et al. (1989) found that about 12 per cent of a sample of IRAS galaxies are Seyferts or LINERs. The interest in studying warm IRAS galaxies has considerably increased with the discovery that many luminous IR galaxies are interacting systems (Soifer et al. 1987; Sanders et al. 1988). Lawrence et al. (1989) found that about $11 \pm 8$ per cent of low-luminosity $\left(<10^{11} L_{\odot}\right)$ and about $46 \pm 12$ per cent of high-luminosity $\left(>10^{11} L_{\odot}\right)$ IRAS galaxies are in interacting systems. Consequently, the interaction could play an important role in producing levels of activity such as those observed in Seyfert 1, Seyfert 2, LINERs, and starburst galaxies.

From the catalogue of AGN candidates (de Grijp et al. 1987) we have selected a sample of southern warm IR galaxies with unknown redshifts (9 out of the 12 galaxies) in order to study their optical spectra, classify them, and search for correlations between optical and IR parameters.

This paper is arranged as follows: in Section 2 we present the observations; in Section 3 we present the spectra and the analysis of their emission lines; in Section 4 we study the classification of the galaxies; in Section 5 we study correlations between optical and far-infrared properties; and in Section 6 we summarize our conclusions.

${ }^{\star}$ Present address: Space Telescope Science Institute, Baltimore, Maryland, USA.

\section{THE OBSERVATIONS}

The observations reported in this work were obtained with the 'Z-machine' (Tonry \& Davis 1979; Latham 1982; da Costa et al. 1984) developed at the Harvard Smithsonian Center for Astrophysics, attached to the Cassegrain spectrograph at the 2.15-m Ritchey-Chrétien telescope of Casleo, San Juan, Argentina, in the period 1989 May to November.

The observations were made using a 600 line $\mathrm{mm}^{-1}$ grating, giving a dispersion of $120 \AA \mathrm{mm}^{-1}$ and covering the wavelength range from 4400 to $7200 \AA$.

The measurements were made through a pair of $3 \times 6$ $\operatorname{arcsec}^{2}$ entrance apertures separated by 37 arcsec on the sky for simultaneous object and sky exposures. The detector employs a dual 936 Reticon system coupled to a high-gain image tube package. A complete object exposure consists of two equal time exposures with the source placed on each of the two channels. In order to obtain reliable pixel-wavelength solutions, comparison exposures were made, just before and after the object. Usually, 50-60 $\mathrm{He}-\mathrm{Ne}-\mathrm{Ar}$ and sky lines were used in a seventh-order polynomial fit of the wavelength solution with typical rms residual of $0.4 \AA$. Incandescent lamp exposures were taken at the end of each night to remove the fixed noise pattern arising in the readout electronics (Latham 1982). The spectra were flux calibrated with stars from the Catalogue 'Southern Spectrophotometric Standards' of Stone \& Baldwin (1983), following standard spectrophotometric reduction techniques. The software package employed in the reductions and in the emission-line analysis has been developed by us at Porto Alegre.

\section{THE SPECTRA}

We estimated the galactic reddening for each object looking for 'neighbouring' objects in the catalogues of Sandage \& 
Tammann (1981), and de Vaucouleurs, de Vaucouleurs \& Corwin (1976). The redshift was calculated by measuring the centroid of a Gaussian centred mainly on the redshifted $[\mathrm{O} \text { III }]_{\lambda 5007}$ line, or on the $\mathrm{H} \alpha$ line whenever the $[\mathrm{O}$ III $]$ line was not available. Figs 1-4 illustrate some of the spectra.

For some of the objects (I0135-1307, I0336-1641, I1834 - 6728 and I1958 - 1818) the stellar population contribution is visible mainly through the overall shape of the continuum, the $\mathrm{Mg}$ I absorption feature, $\mathrm{H} \beta$ in absorption and the $\mathrm{TiO}_{\lambda 6290}$ absorption band (see Fig. 3 as an example of this method of stellar population subtraction applied to I1958-1818). It is important to take the stellar population contribution into account in order to isolate the emission from and analyse the gas in these galaxies. We followed the stellar population synthesis method of Bica (1988) and found that the S2 red template represents quite well the stellar population in the above objects. This template is an average of spiral galaxies, attaining a maximum metallicity $[Z]$ $\left.Z_{\odot}\right]=+0.3$ and dominated by the old population with age $>5 \times 10^{9} \mathrm{yr}$, with the strongest flux contribution, 87 per cent at $5870 \AA$ coming from components which are globular clusters. A further reddening correction was applied to I0336-1641 and I1834-6728 so that the continua of the template and those of the observed spectra matched. For further details and examples of this method of stellar population synthesis, see Bica (1988) and Bonatto, Bica \& Alloin (1989).

In Table 1 we present the identification of each object along with its number according to de Grijp et al. (1987). We also give the redshift $z$, the galactic colour-excess $E(B-V)_{\mathrm{G}}$, and the additional reddening correction $E(B-V)_{\mathrm{P}}$, for the four objects mentioned above. In column 6 of Table 1 we give the spectral classification of the galaxies according to Section 4; and in the last column we list the log of the far-infrared luminosity (relative to the $\operatorname{Sun}) \log \left(L_{\mathrm{IR}} / L_{\odot}\right)$, where $\log \left(L_{\mathrm{IR}}\right)=\log \left(F_{\mathrm{IR}}\right)+2 \log [z(1+z)]+57.28$, and $F_{\text {IR }}=1.26 \times 10^{-11}\left(2.58 S_{60}+S_{100}\right) \mathrm{erg} \mathrm{s}^{-1} \mathrm{~cm}^{-2}$, where $S_{60}$ and $S_{100}$ are the flux densities at 60 and $100 \mu \mathrm{m}$ respectively. The S2 red template, when subtracted, leaves the $\mathrm{H} \alpha$ flux nearly unaffected. So we can use the non-subtracted $\mathrm{H} \alpha$ flux in order to scale the other lines (affected by the template

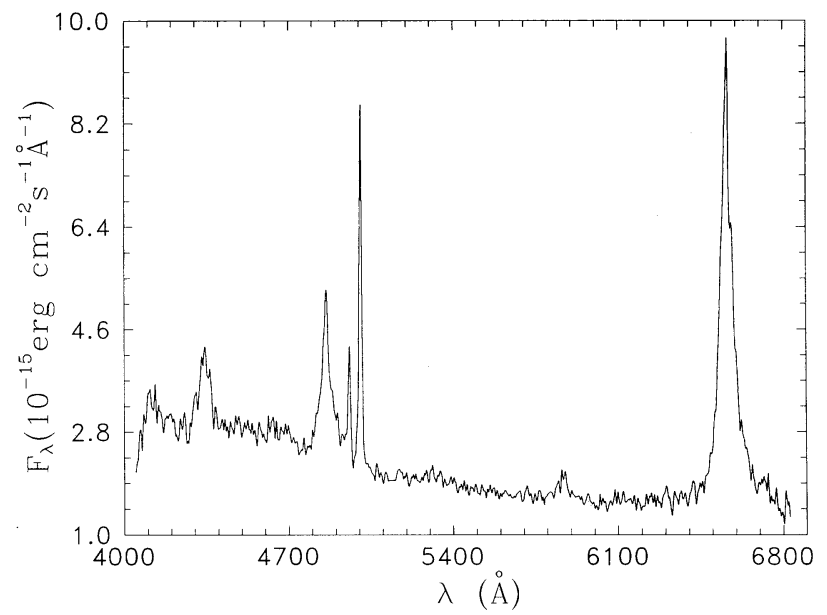

Figure 1. The reddening-corrected and flux-calibrated spectrum of the IRAS Seyfert 1 galaxy I0137-2230 in the range $4050-6850 \AA$ A. Spectral classification according to Section 4.

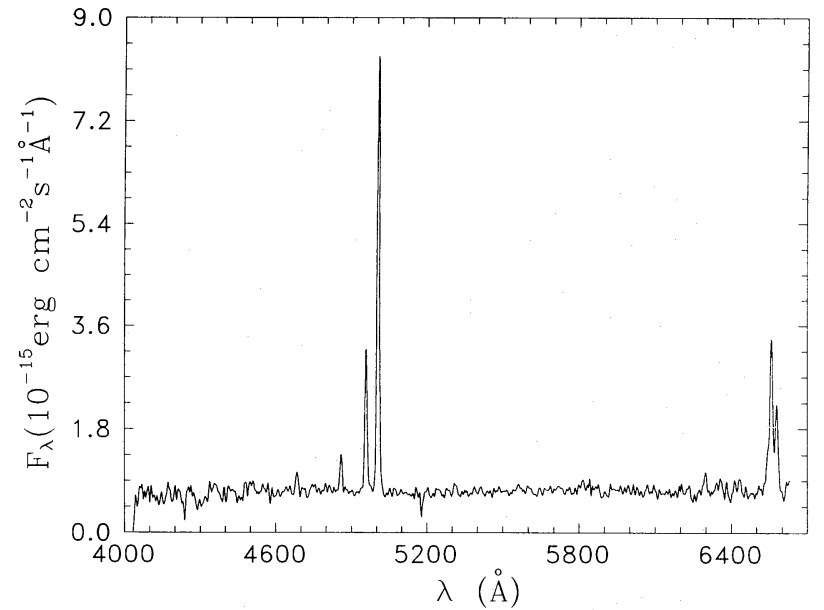

Figure 2. Same as Fig. 1 for the IRAS Seyfert 2 galaxy I0310 - 5131 .

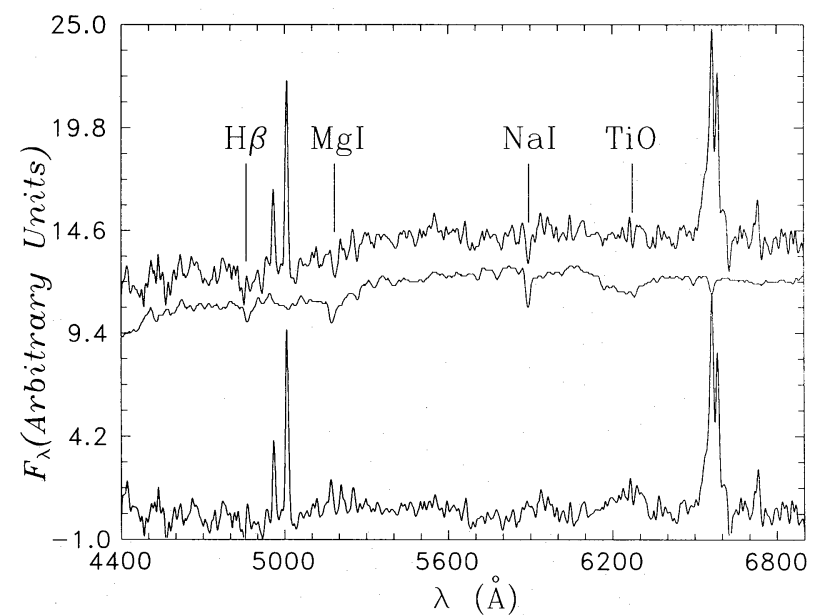

Figure 3. The spectrum of the IRAS Seyfert 2 galaxy I1958-1818 is shown before (top panel) and after (bottom panel) subtraction of the stellar population template S2 (middle panel). See the text for more details. Ordinate in arbitrary units. Abscissa in $\AA$. Outstanding absorption features are indicated.

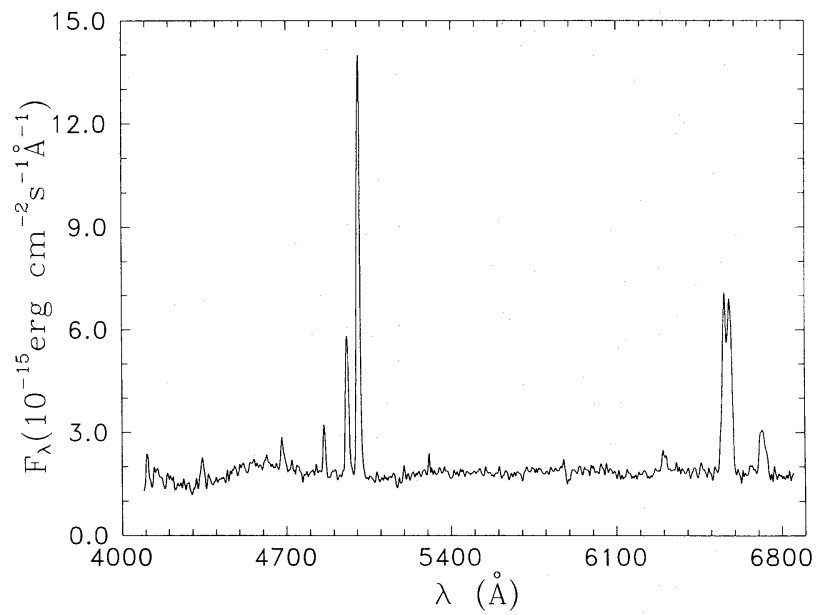

Figure 4. Same as Fig. 1 for the IRAS Seyfert 2 galaxy I $2055-5211$. 
subtraction due to template absorption features) to absolute fluxes.

After correcting for the galactic reddening and the redshift, and after the subtraction of the stellar population from the objects that needed it, we fitted Gaussians to the remaining absorption-free emission lines. Examples of this procedure can be seen in Figs 5 and 6 . The results for the objects with a single component in $\mathrm{H} \alpha$ are presented in Table 2, where we give the total flux, the error in the flux $(\sigma$, as estimated from the noise level around each line), and the FWHM. The same result for the $\mathrm{H} \alpha$ multicomponent objects are listed in Table 3. Objects marked with an asterisk (*) have had the stellar population subtracted. Our fitting procedures were always constrained to the theoretical ratios $[\mathrm{O} \text { III }]_{\lambda 4959}=\frac{1}{3}[\mathrm{O} \text { III }]_{\lambda 5007}$, and $\left[\mathrm{N} \mathrm{III}_{\lambda 6548}=\frac{1}{3}[\mathrm{~N} \mathrm{II}]_{\lambda 6584}\right.$, so these two lines are not listed in Tables 2 and 3. The penultimate line of Tables 2 and 3 gives the ratio $\mathrm{H} \alpha / \mathrm{H} \beta$. For those objects with composed $\mathrm{H}$ i Balmer profiles, we calculate the Balmer decrement by summing the flux of all components.

The Balmer decrement in Tables 2 and 3 indicates the presence of dust in the line-emitting regions of these galaxies. We assume that the optical properties of the dust are identical with those of dust in our Galaxy and, for lack of other lines, we use only the measured ratio $\mathrm{H} \alpha / \mathrm{H} \beta$ to determine the amount of internal extinction. Following Veilleux \& Osterbrock (1987, hereafter VO87), we also assume an intrinsic ratio $\mathrm{H} \alpha / \mathrm{H} \beta=3.1$ for our objects because, as it will be seen later (Section 4), all of our galaxies can be considered as AGNs, and collisional excitation plays an important role in determining the emission-line spectrum in such objects. The internal reddening correction was determined from the interstellar extinction curves given by Seaton (1979) and the expression $F_{0}(\lambda)=F(\lambda) 10^{\left[0.4 E(B-V)_{1} \chi(x)\right]}$, where $F_{0}(\lambda)$ and $F(\lambda)$ are, respectively, the corrected and the measured fluxes at $\lambda$, $\chi(x)$ is the extinction function, and $x=1 / \lambda$, with $\lambda$ expressed in $\mu \mathrm{m} . \chi(x)$ was expressed as a third-order polynomial fitted to the points of $\chi(x)$ given by Nandy et al. (1975). The corrected flux values are given in Tables 2 and 3 in the line below the measured flux for each emission line, and in the last line of Tables 2 and 3 we list the 'internal' colour excess $E(B-V)_{1}$.

Our sample contains three objects (I1249-1308, I1548-0344 and I1958-1818) in common with the sample of OR85. For I1958-1818 our $\mathrm{H} \alpha$ flux is in very good agreement with the value quoted by OR85, although

Table 1. Details of the objects.

$\begin{array}{ccccccc}\text { IRAS } & \mathrm{N} & \mathrm{z} & \mathrm{E}(\mathrm{B}-\mathrm{V})_{G} & \mathrm{E}(\mathrm{B}-\mathrm{V})_{P} & \text { Class. } & \log \left(L_{I R} / L_{\odot}\right) \\ 0019-7926 & 006 & 0.07289 & 0.02 & - & \mathrm{S} 2 & 11.62 \\ 0135-1307 & 030 & 0.04050 & 0.00 & 0.00 & \mathrm{~S} 2 & 10.61 \\ 0137-2230 & 031 & 0.08657 & 0.00 & - & \mathrm{S} 1 & 11.11 \\ 0310-5131 & 076 & 0.07823 & 0.00 & - & \mathrm{S} 2 & 11.01 \\ 0336-1641 & 098 & 0.03735 & 0.00 & 0.30 & \mathrm{~S} 2 & 10.76 \\ 1249-1308 & 309 & 0.01418 & 0.00 & - & \mathrm{S} 1.5 & 9.84 \\ 1258-3039 & 312 & 0.01633 & 0.04 & - & \mathrm{S} 2 & 9.91 \\ 1548-0344 & 383 & 0.03095 & 0.03 & - & \mathrm{S} 2 & 10.66 \\ 1834-6728 & 472 & 0.01452 & 0.07 & 0.10 & \mathrm{~S} 2 & 10.03 \\ 1958-1818 & 495 & 0.03730 & 0.13 & 0.00 & \mathrm{~S} 2 & 10.52 \\ 2020-5635 & 502 & 0.06011 & 0.03 & - & \mathrm{S} 1.9 & 10.86 \\ 2055-5211 & 513 & 0.05073 & 0.02 & - & \mathrm{S} 2 & 10.90\end{array}$

line-ratios differ somewhat; probably the differences can be ascribed to OR85 not having taken into account the stellar population in this object; for I1548-0344 our fluxes agree with those of OR85 to within 15 per cent; the differences are important for I1249-1308, but this object presents a composed $\mathrm{H} \alpha$ profile, so that the differences may be attributed to the deblending procedures.

\section{SPECTRAL CLASSIFICATION}

\subsection{The optical data}

In the past, several attempts have been made to classify the spectra of extragalactic objects according to the principal excitation mechanisms. Usually, diagnostic diagrams with emission-line ratios of easily-observed lines are employed to achieve this goal. In this section we apply some diagnostic diagrams to our galaxies and classify them.

A first inspection of the data regarding the three galaxies in Table 3 (and Fig. 1 for I0137-2230) shows us that I0137-2230 is a classical Seyfert 1 galaxy with $\mathrm{H}_{\text {I }}$ Balmer lines $\left(F W H M \geq 4000 \mathrm{~km} \mathrm{~s}^{-1}\right)$ much broader than the for-

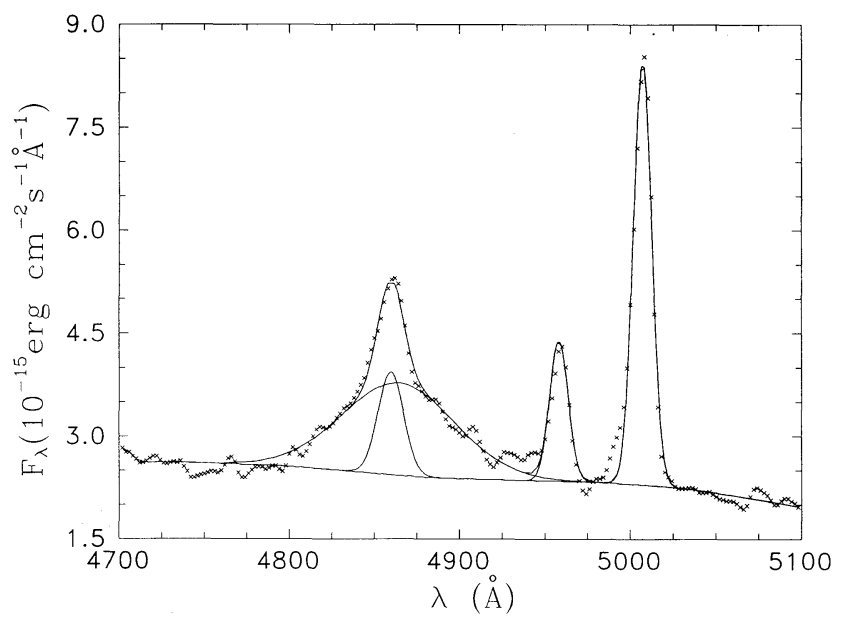

Figure 5. Gaussian fit for the $\mathrm{H} \beta+\left[\mathrm{O}_{\mathrm{III}}\right]_{\lambda \lambda 4959,5007}$ profile for the IRAS Seyfert 1 galaxy $10137-2230$.

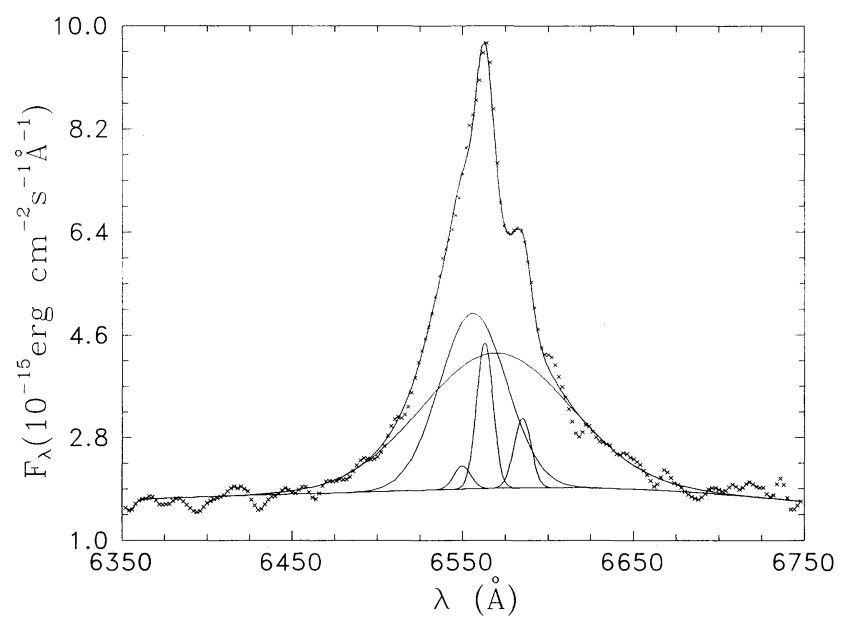

Figure 6. Same as Fig. 5 for the $\mathrm{H} \alpha+\left[\mathrm{N}_{\mathrm{II}}\right]_{\lambda \lambda 6548,83}$ profile for the IRAS Seyfert 1 galaxy I0137-2230. 
Table 2. IRAS Seyfert 2 emission-line parameters.

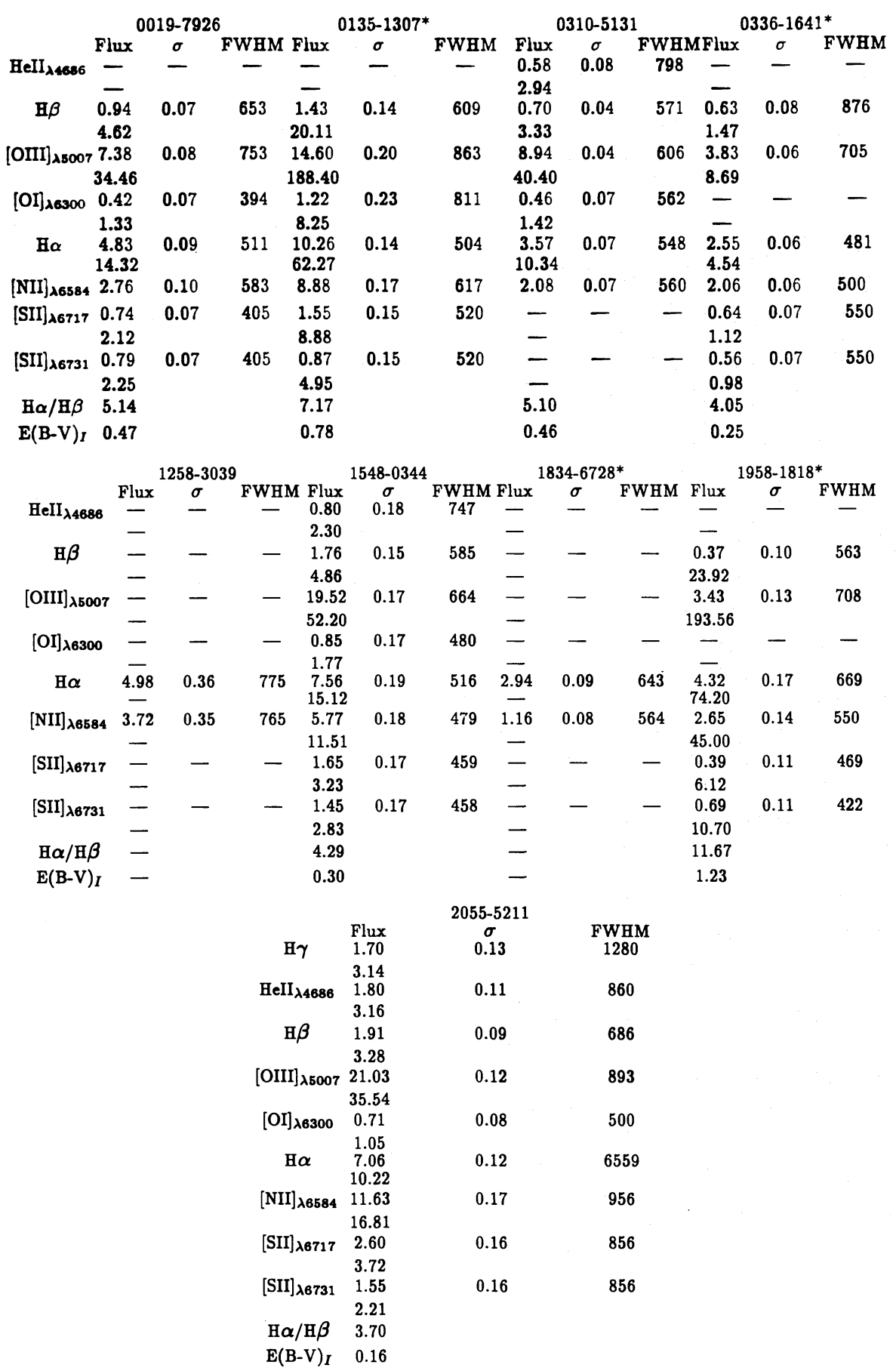

Notes: *Stellar population-subtracted. Additional features. I1548-0344: $[\mathrm{Fe} \text { VII }]_{\lambda 6087}$ Flux $=1.20 \pm 0.2, \mathrm{FWHM}=567,[\mathrm{Fe} \mathrm{x}]_{\lambda 6375}$ Flux $=0.58 \pm 0.14, \mathrm{FWHM}=378 ; \mathrm{I} 2055-5211$ : $\mathrm{H} \delta$ Flux $=1.21 \pm 0.14, \mathrm{FWHM}=802,[\mathrm{~S} \text { III }]_{\lambda 6312}$ Flux $=0.41 \pm 0.07, \mathrm{FWHM}=390$. Flux and $\sigma$ in $10^{-14} \mathrm{erg} \mathrm{cm}^{-2} \mathrm{~s}^{-1}$, FWHM in $\mathrm{km} \mathrm{s}^{-1}$.

bidden lines (FWHM $\leq 700 \mathrm{~km} \mathrm{~s}^{-1}$ ), clearly having a strong contribution of a power-law to the continuum of this galaxy; I1249-1308 is classified as a Seyfert 1 by OR 85 but, based on our observations and on the width of its emission lines (FWHM $<2000 \mathrm{~km} \mathrm{~s}^{-1}$ for the $\mathrm{H}$ I Balmer lines), we classify this galaxy as an intermediate type, between Seyfert 1 and Seyfert 1.5; finally, I2020 - 5635 has characteristics close to the definition (according to OR85) of a Seyfert 1.9.
Veuilleux \& Osterbrock (1987) present diagnostic diagrams involving the line-ratios $[\mathrm{O} \mathrm{III}]_{\lambda 5007} / \mathrm{H} \beta,[\mathrm{N} \mathrm{II}]_{\lambda 6583} /$ $\mathrm{H} \alpha,\left[\mathrm{S} \mathrm{II}_{\lambda \lambda 6717,31} / \mathrm{H} \alpha\right.$, and $[\mathrm{O} \mathrm{I}]_{\lambda 6300} / \mathrm{H} \alpha$, which enable one to distinguish between narrow-line active galaxies and $\mathrm{H}$ II region-like galaxies. These line ratios are nearly unaffected by the reddening corrections and errors in the flux calibration. In the VO87 diagnostic diagrams we have plotted the reddening-corrected line ratios measured in our objects, and 
Table 3. IRAS multi-component galaxies.

\begin{tabular}{|c|c|c|c|c|c|c|c|c|c|}
\hline & & $37-223$ & & & $49-1308$ & & & $20-563$ & \\
\hline $\mathrm{H} \gamma$ & $\begin{array}{l}\text { Flux } \\
6.69\end{array}$ & $\begin{array}{c}\sigma \\
0.63\end{array}$ & $\underset{3622}{\text { FWHM }}$ & $\begin{array}{l}\text { Flux } \\
9.39\end{array}$ & $\begin{array}{c}\sigma \\
0.24\end{array}$ & $\underset{1406}{\text { FWHM }}$ & Flux & $\underline{\sigma}$ & $\begin{array}{c}\text { FWHM } \\
-\end{array}$ \\
\hline & 6.69 & & & 12.28 & & & - & & \\
\hline$[\mathrm{OIII}]_{\lambda 4363}$ & 0.39 & 0.11 & 645 & 1.93 & 0.19 & 1100 & - & 一 & - \\
\hline & 0.39 & & & 2.51 & & & - & & \\
\hline $\mathrm{Hell}_{\boldsymbol{\lambda} 4686}$ & - & 一 & - & $\begin{array}{l}1.29 \\
1.65\end{array}$ & 0.18 & 669 & - & - & - \\
\hline$(\mathrm{H} \beta)_{B}$ & 11.54 & 0.58 & 5155 & 15.00 & 0.43 & 1552 & - & - & - \\
\hline & 11.54 & & & 19.01 & & & - & & \\
\hline$(\mathrm{H} \beta)_{N}$ & 3.04 & 0.13 & 1111 & - & - & - & 1.00 & 0.07 & 963 \\
\hline & 3.04 & & & - & & & 12.28 & & \\
\hline$[\mathrm{OIII}]_{\lambda 5007}$ & 8.39 & 0.08 & 723 & 29.93 & 0.21 & 735 & 14.36 & 0.06 & 857 \\
\hline & 8.39 & & & 37.65 & & & 162.52 & & \\
\hline$[\mathrm{OI}]_{\lambda 6300}$ & - & - & 一 & 1.21 & 0.20 & 681 & 0.62 & 0.08 & 579 \\
\hline & - & & & 1.44 & & & 3.80 & & \\
\hline$(\mathrm{H} \alpha)_{B}$ & 27.47 & 0.82 & 4678 & 39.08 & 0.55 & 1805 & 4.45 & 0.33 & 2161 \\
\hline & 27.47 & & & 45.94 & & & 24.62 & & \\
\hline$(\mathrm{H} \alpha)_{I}$ & 14.69 & 0.37 & 2108 & - & - & - & - & - & - \\
\hline & 14.69 & & & - & & & 一 & & \\
\hline$(\mathrm{H} \alpha)_{N}$ & 3.26 & 0.09 & 513 & 11.06 & 0.14 & 450 & 2.43 & 0.07 & 472 \\
\hline$[\mathrm{NIII}]_{\lambda 6584}$ & $\begin{array}{l}3.26 \\
1.57\end{array}$ & 0.09 & 532 & $\begin{array}{c}13.00 \\
9.80\end{array}$ & 0.14 & 456 & $\begin{array}{c}13.44 \\
2.35\end{array}$ & 0.08 & 554 \\
\hline & 1.57 & & & 11.51 & & & 12.91 & & \\
\hline$[\mathrm{SII}]_{\lambda 6717}$ & - & - & - & 2.94 & 0.14 & 445 & 1.08 & 0.07 & 453 \\
\hline & - & & & 3.44 & & & 5.64 & & \\
\hline$[\mathrm{SII}]_{\lambda 6731}$ & - & - & 一 & 2.72 & 0.13 & 409 & 0.78 & 0.07 & 453 \\
\hline & - & & & 3.18 & & & 4.07 & & \\
\hline $\mathrm{H} \alpha / \mathrm{H} \beta$ & 3.11 & & & 3.34 & & & 6.88 & & \\
\hline $\mathrm{E}(\mathrm{B} \cdot \mathrm{V})_{I}$ & 0.00 & & & 0.07 & & & 0.74 & & \\
\hline
\end{tabular}

Notes: B, I and $\mathrm{N}$ refer to the Broad, Intermediate and Narrow components. Additional features. I0137-2230: $\mathrm{He}_{25876}$ Flux $=2.59 \pm 0.21$, FWHM= 2613; flux and $\sigma$ in $10^{-14} \mathrm{erg} \mathrm{cm}^{-2} \mathrm{~s}^{-1} ; \mathrm{FWHM}$ in $\mathrm{km} \mathrm{s}^{-1}$.

in Fig. 7 we illustrate this with the diagnostic diagram $\left[\mathrm{O} \mathrm{III}_{\lambda 5007} / \mathrm{H} \beta\right.$ versus $[\mathrm{N} \mathrm{II}]_{\lambda 6583} / \mathrm{H} \alpha$. In this figure the area delimited by the solid line is occupied by Seyfert $2 \mathrm{~s}$ and narrow-line radio galaxies (NLRG), objects photo-ionized by a 'non-thermal' or 'power-law' continuum; the short-dashed curve encloses $\mathrm{H}$ II regions, $\mathrm{H}$ II regions in a nucleus, starburst galaxies, and $\mathrm{H}$ II galaxies, objects photo-ionized by hot stars; and the long-dashed curve encloses the LINERs. We also have included the narrow components of $\mathrm{H} \alpha$ and $\mathrm{H} \beta$ for the three objects of Table 3 in Fig. 7.

The three diagnostic-diagrams give essentially the same results: all the objects listed in Table 2 (except I1258-3039 and $\mathrm{I} 1834-6728$ which have no detectable $[\mathrm{O} \mathrm{III}]_{\lambda 5007}$ and $\left.\left[\mathrm{S}_{\mathrm{II}}\right]_{\lambda \lambda 6717,31}\right)$ fall within the area occupied by the Seyfert $2 \mathrm{~s}$ and NLRGs. I2020 - 5635, previously classified as Seyfert 1.9 , also lies in the Seyfert 2 area; the narrow components of I0137-1308 and of I1249-1308 are located in the region of the active galaxies, halfway between the Seyfert $2 \mathrm{~s}$ and LINERs. The narrow-line region of these two galaxies are still photo-ionized by a power-law continuum but, apparently, there is also a considerable contribution of photoionization by hot stars.

A similar classification has been obtained using the BPT (Baldwin, Phillips \& Terlevich 1981) diagnostic-diagram involving $[\mathrm{O} \text { III }]_{\lambda 5007} / \mathrm{H} \beta$ versus $[\mathrm{N} \mathrm{III}]_{\lambda 6583} / \mathrm{H} \alpha$.

\subsection{The infrared data}

Far-infrared flux-densities in the IRAS bands at 12, 25, 60 and $100 \mu \mathrm{m}$ were taken from the IRAS point source catalog (1985). Since the galaxies of our sample have a redshift $z>0.014$, corresponding to a radial velocity $\left(H=50 \mathrm{~km} \mathrm{~s}^{-1}\right.$ $\left.\mathrm{Mpc}^{-1}\right) V_{\mathrm{R}}>4000 \mathrm{~km} \mathrm{~s}^{-1}$, we should not expect flux losses

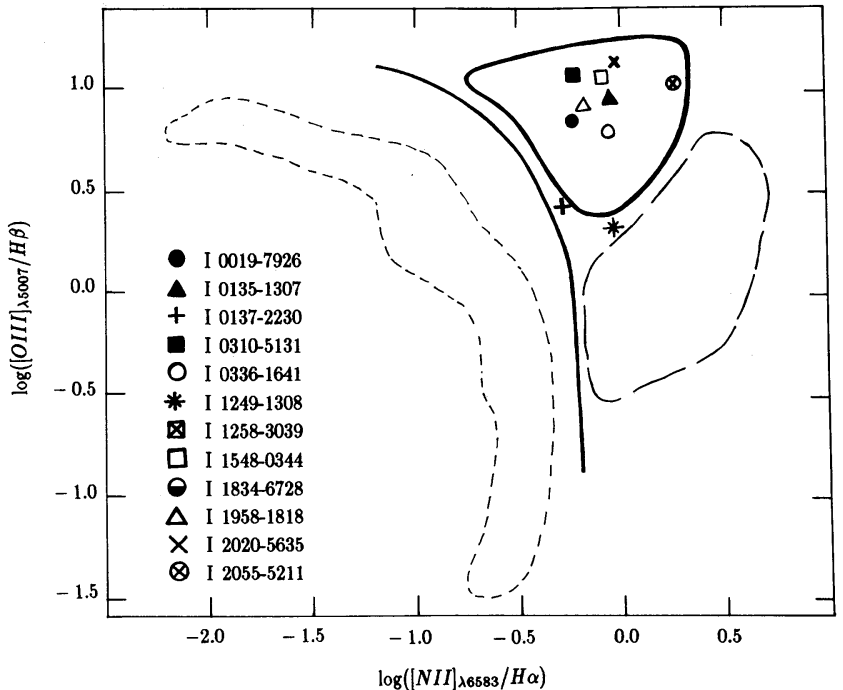

Figure 7. The reddening-corrected line ratios (see Tables 2 and 3 ) $\log \left([\mathrm{O} \mathrm{III}]_{\lambda 5007} / \mathrm{H} \beta\right)$ versus $\log \left([\mathrm{N} \mathrm{II}]_{\lambda 6583} / \mathrm{H} \alpha\right)$ are plotted in this diagnostic diagram (adapted from VO87). The inset shows the symbols that identify each object on the plot. The short-dashed line defines the locus of the $\mathrm{H}$ II regions, starburst galaxies, and $\mathrm{H}$ II galaxies; the long-dashed line encloses the LINERs; and the continuous line encloses the Seyfert 2 and the narrow-line radio galaxies. For I0137 - 2230 and I1249 - 1308 only the narrow components of the Balmer lines are used in the line ratios.

due to different diaphragm sizes at $25 \mu \mathrm{m}(\sim 0.76 \times 4.6$ $\left.\operatorname{arcmin}^{2}\right), 60 \mu \mathrm{m}\left(\sim 1.5 \times 4.75 \operatorname{arcmin}^{2}\right)$, and $100 \mu \mathrm{m}$ $\left(\sim 3.0 \times 5.0 \operatorname{arcmin}^{2}\right)$.

The objects of our sample were chosen because of their relatively high IR luminosity $\left(9.84<\log \left(L_{\mathrm{IR}} / L_{\odot}\right)<11.62\right)$, 
thus we can use the far-infrared colours of these galaxies as another criterion to classify our objects. To do this we use the far-infrared colour-colour diagnostic diagrams of Sekiguchi (1987). Far-infrared colours are calculated by taking the logarithmic ratios of the flux densities at two IRAS bands, thus $-\alpha(\lambda 1, \lambda 2)=\log \left(S_{\lambda 1} / S_{\lambda 2}\right) / \log (\lambda 1 / \lambda 2)$, where $S_{\lambda}$ is the flux-density (in Jy) at $\lambda$, and $\lambda 1>\lambda 2$. As with the diagrams of VO87, we also have marked off regions occupied by specific classes of objects. Thus, non-active spiral galaxies are enclosed by the short-dashed curve, and starburst galaxies are enclosed by the dot-dashed curve. Seyfert 2 galaxies are scattered on the diagram above the non-active spiral galaxies region (Fig. 8). Four objects (I0137-2230, I0336-1641, I1548-0344 and I2020-5635) have only upper limit detections at $100 \mu \mathrm{m}$, indicated by arrows in Fig. 8 .

Several of our objects in the adapted far-infrared colour-colour diagram (Fig. 8) lie close to the power-law curve, the exceptions being I1548 - 0344, I0336 - 1641 and I0137-2230 (upper limits at $100 \mu \mathrm{m}$ ) on the left of the PL curve, and I0019-7926 and I2055-5211 on the right of the PL curve.

The galaxies I0019-7926 and I2055-5211 which are clearly classified as Seyfert 2 through the optical data (Section 4a), are located in the far-infrared colour-colour diagram near the blackbody model curve with their blackbody temperatures higher than the temperatures of the starburst galaxies. This apparent contradiction can be interpreted as a combination of two mechanisms that excite the dust: basically a power-law continuum source covered by gas and dust clouds in which massive star formation could be happening (Sekiguchi 1987). These clouds re-radiate the power-law photons with a blackbody spectrum. It is interesting to note that I0019-7926 is the most IR-luminous galaxy of our sample, with $\log \left(L_{\mathrm{IR}} / L_{\odot}\right)=11.62$, and also that this object is an interacting galaxy.

\section{OPTICAL VERSUS FAR-INFRARED LUMINOSITIES}

In this section we investigate how the luminosities of the farinfrared IRAS bands correlate with the luminosities of

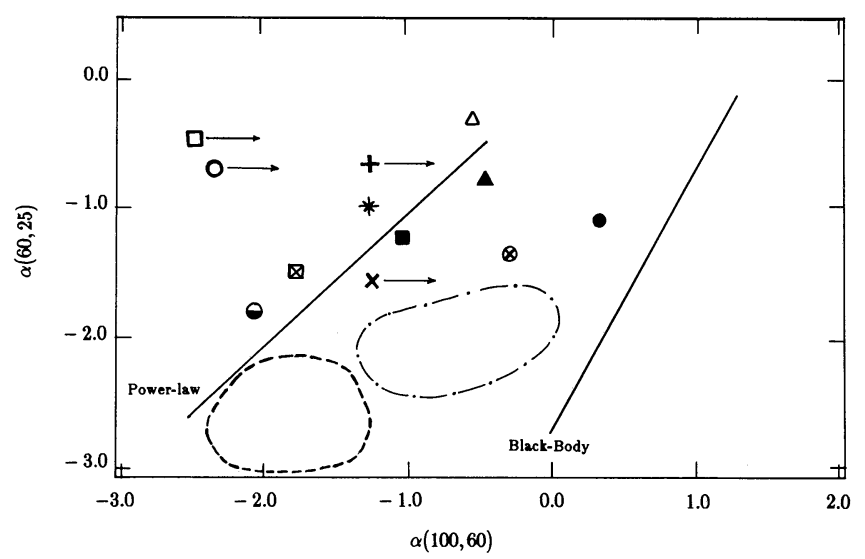

Figure 8. Far-infrared $\alpha(60,25)$ versus $\alpha(100,60)$ colour-colour diagnostic diagram. Objects labelled as in Fig. 7. Short-dashed line encloses normal (non-active) spiral galaxies, and the dot-dashed line encloses the Starburst Galaxies. Pure power-law $\left(F_{v} \propto \nu^{\alpha}\right)$ and onetemperature blackbody models are indicated. Arrows indicate objects with upper limits at $100 \mu \mathrm{m}$. optical lines. This issue has been studied by Dultzin-Hacyan, Moles \& Masegosa (1988) who found that, for all Seyfert 2 galaxies in the Véron-Cetty \& Véron (1987) catalogue, there is a strong correlation between the luminosity at $25 \mu \mathrm{m}$ and the nuclear $\mathrm{H} \beta$ luminosity, thus suggesting that the bulk of the $25-\mu \mathrm{m}$ emission has the same origin (nuclear) as the $\mathrm{H} \beta$ emission; for the other IRAS bands they have found weak or no correlation.

In our galaxy sample, 100 per cent of the objects have $\mathrm{H} \alpha$ measurable, so we use this line to search for correlations. We have plotted in Fig. 9 the luminosity at $60 \mu \mathrm{m}$ against the $\mathrm{H} \alpha$ luminosity (relative to the Sun). The straight line in the plot shows the result of a linear least-squares fit performed on the data. The best correlation (with multiple linear-correlation coefficient $R^{2}=0.78$ ) was found for the $25-\mu$ m luminosity; next comes the $60-\mu \mathrm{m}$ luminosity with $R^{2}=0.72$, and finally the $100-\mu \mathrm{m}$ luminosity with $R^{2}=0.63$ (we have excluded from this last fit the four objects with upper limits at 100 $\mu \mathrm{m})$. Thus we can say that both the $25-\mu \mathrm{m}$ and the $60-\mu \mathrm{m}$ emissions have the same origin as $\mathrm{H} \alpha$ and, to a lesser extent, the same can be said of the $100-\mu$ m emission.

Another good correlation was found between the farinfrared luminosity $L_{\mathrm{IR}}$ and $\mathrm{H} \alpha$, with $R^{2}=0.69$. This is shown in Fig. 10 where we plot the far-infrared luminosity $\log \left(L_{\mathrm{IR}} / L_{\odot}\right)$ against the $\log \left(L_{\mathrm{H} \alpha} / L_{\odot}\right)$ luminosity.

Concerning the ratio $L_{60} / L_{\mathrm{H} \alpha}$ we find that, for the Seyfert $2 s$ (including the Seyfert 1.9 I2020-5635) of our sample, $400<L_{60} / L_{\mathrm{H} \alpha}<3000$, whereas for the Seyfert 1.5 I1249-1308 $L_{60} / L_{\mathrm{H} \alpha} \approx 120$, and for the Seyfert 1 I0137-2230 $L_{60} / L_{\mathrm{H} \alpha} \approx 60$. This suggests a strong thermal component in the IRAS Seyfert $2 \mathrm{~s}$ which is less conspicuous in the Seyfert 1s. Leech et al. (1989) presented a model with two kinds of dust clouds (type I with $A_{V} \approx 20$, and type II with $A_{V} \approx 1$ ). In this picture, objects with large $L_{60} / L_{\mathrm{H} \alpha}$ $(>5000)$ are the youngest starbursts, dominated by type I clouds. Plotting our data in Fig. 9 of Leech et al. (1989) we see that the IR luminosity of the Seyfert 2 galaxies of our sample is still dominated by young star-forming regions, but there is also a significant contribution of type II clouds (lower optical depth).

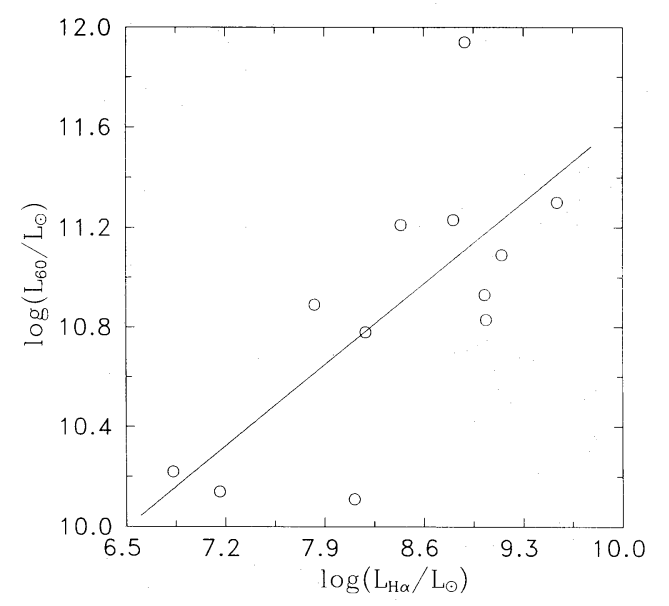

Figure 9. The luminosity of the IRAS band at $60 \mu \mathrm{m} \log \left(L_{60} / L_{\odot}\right)$, plotted against the $\mathrm{H} \alpha$ luminosity (relative to the $\operatorname{Sun}$ ), $\log \left(L_{\mathrm{H} \alpha} / L_{\odot}\right)$. The multiple linear correlation coefficient is $R^{2}=0.72$. 


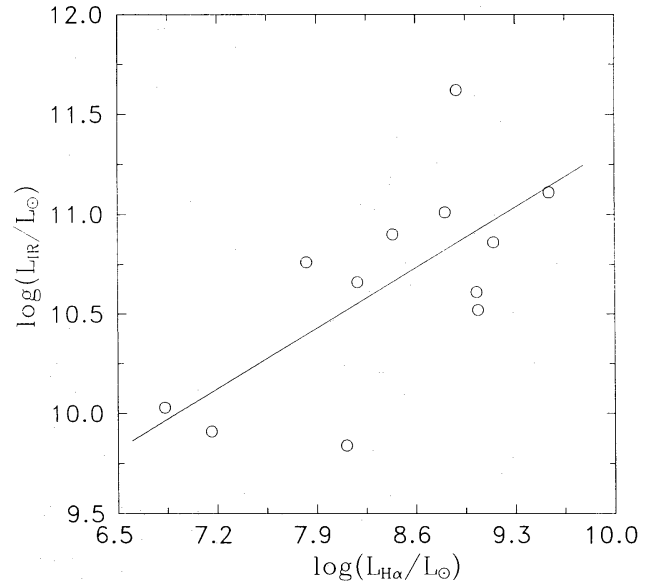

Figure 10. The far-infrared luminosity $L_{\mathrm{IR}}$ is plotted against the $\mathrm{H} \alpha$ luminosity showing a correlation between these two quantities $\left(R^{2}=0.69\right)$

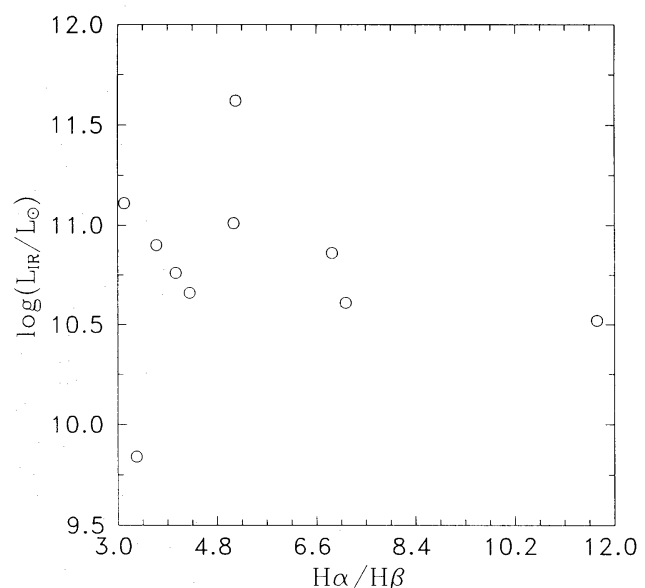

Figure 11. The far-infrared luminosity $L_{\mathrm{IR}}$ shows no correlation with the Balmer decrement $\mathrm{H} \alpha / \mathrm{H} \beta$, thus implying that the dust content in the IRAS galaxies is not the main factor determining the far-IR luminosity.

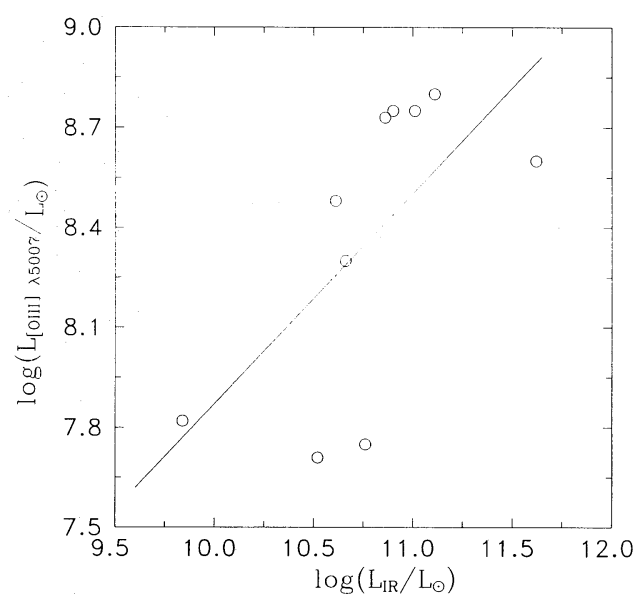

Figure 12. This figure shows the correlation $\left(R^{2}=0.66\right)$ between the far-infrared luminosity $L_{\mathrm{IR}}$ and the $[\mathrm{O} \mathrm{III}]_{\lambda 5007}$ luminosity, thus suggesting that $L_{\mathrm{IR}}$ depends strongly on the intensity of the ionizing source.
Assuming that the ratio $\mathrm{H} \alpha / \mathrm{H} \beta$ gives a measure of the amount of dust present in the line-emitting regions of active galaxies, we searched for a link between the dust and the farinfrared luminosity. This can be seen in Fig. 11 in which we have plotted $\log \left(L_{\mathrm{IR}} / L_{\odot}\right)$ versus $\mathrm{H} \alpha / \mathrm{H} \beta$. It is apparent that there is no correlation between these two quantities. On the other hand, we have found a correlation between $L_{\mathrm{IR}}$ and the $[\mathrm{O} \mathrm{III}]_{\lambda 5007}$ luminosity $\left(R^{2}=0.66\right.$, Fig. 12). One way to interpret these two facts is to assume that the far-infrared luminosity depends more strongly on the intensity of the central ionizing source (i.e. on the number of ionizing photons) than on the amount of dust, if recombination is the only mechanism responsible for the Balmer lines.

\section{CONCLUSIONS}

In this paper we have analysed 12 southern warm IRAS galaxies. Using optical and far-infrared colour-colour diagnostic diagrams, we have classified these galaxies according to their principal excitation mechanism and found that all are Seyfert galaxies.

There exist very good correlations between the luminosity at 25 and $60 \mu \mathrm{m}$ and the $\mathrm{H} \alpha$ luminosity, thus suggesting that the emission at these two IRAS bands has the same origin as the $\mathrm{H} \alpha$ emission; also the far-infrared luminosity $L_{\mathrm{IR}}$ correlates well with $\mathrm{H} \alpha$.

On the assumption that recombination is the only mechanism responsible for the Balmer lines and that $\mathrm{H} \alpha / \mathrm{H} \beta$ is a measure of the dust content, the far-infrared luminosity is more dependent on the number of ionizing photons than on the amount of dust.

Another point which should be stressed is that I0019-7926, the IR-brightest galaxy of our sample is an interacting galaxy.

\section{ACKNOWLEDGMENTS}

$\mathrm{ChB}$ and MGP acknowledge financial support by the Brazilian institution CNPq; SL expresses his gratitude to the staff members and observing assistants of CASLEO Observatory. Special thanks are also due to Dr C. Wilmer and Mr M. Rizo for their help in the data processing.

\section{REFERENCES}

Baldwin, J. A., Phillips, M. M. \& Terlevich, R., 1981. Publs astr. Soc. Pacif., 93, 5 (BPT).

Bica, E., 1988. Astr. Astrophys., 195, 76.

Bonatto, Ch. J., Bica, E. \& Alloin, D., 1989. Astr. Astrophys., 226, 23.

da Costa, L. N., Pellegrini, P. S., Nunes, M. A., Willmer, C. \& Latham, D. W., 1984. Astr. J., 89, 1310.

de Grijp, M. H. K., Miley, G. K. \& Lub, J., 1987. Astr. Astrophys. Suppl. Ser., 70, 95.

de Grijp, M. H. K., Miley, G. K., Lub, J. \& de Jong, T., 1985. Nature, 314, 240.

de Vaucouleurs, G., de Vaucouleurs, A. \& Corwin, H. G., 1976. Second Reference Catalogue of Bright Galaxies, University of Texas Press, Austin.

Dultzin-Hacyan, D., Moles, M. \& Masegosa, J., 1988. Astr. Astrophys., 206, 95.

IRAS Point Source Catalog, 1985. Joint IRAS Science Working Group, US Government Printing Office, Washington, DC. 
Latham, D. W., 1982. Instrumentation For Astronomy With Very Large Optical Telescopes, IAU Colloq. No. 67, p. 259, ed. Humphries, C. M., Reidel, Dordrecht.

Lawrence, A., Rowan-Robinson, M., Leech, K., Jones, D. H. P. \& Wall, J. V., 1989. Mon. Not. R. astr. Soc., 240, 329.

Leech, K. J., Penston, M. V., Terlevich, R., Lawrence, A., RowanRobinson, M. \& Crawford, J., 1989. Mon. Not. R. astr. Soc., 240, 349.

Miley, G. K., Neugebauer, G. \& Soifer, B. T., 1985. Astrophys. J., 293, L11.

Nandy, K., Thompson, G. I., Jamar, C., Monfils, A. \& Wilson, R., 1975. Astr. Astrophys., 44, 195.

Osterbrock, D. E. \& de Robertis, M. M., 1985. Publs astr. Soc. Pacif., 97, 1129.

Sandage, A. \& Tammann, G. A., 1981. A Revised Shapley-Ames
Catalogue of Bright Galaxies, Carnegie Institution, Washington, DC.

Sanders, D. B., Soifer, B. T., Elias, J. H., Madore, B. F., Mathews, K., Neugebauer, G. \& Scoville, N. Z., 1988. Astrophys. J., 325, 74.

Seaton, M. J., 1979. Mon. Not. R. astr. Soc., 187, 73P.

Sekiguchi, K., 1987. Astrophys. J., 316, 145.

Soifer, B. T., Sanders, D. B., Madore, B. F., Neugebauer, G., Persson, C. J., Persson, S. E. \& Rice, W. L., 1987. Astrophys. J., 320, 238.

Stone, R. P. S. \& Baldwin, J. A., 1983. Mon. Not. R. astr. Soc., 204, 357.

Tonry, J. \& Davis, M., 1979. Astr. J., 84, 1511.

Veilleux, S. \& Osterbrock, D. E., 1987. Astrophys. J. Suppl., 63, 295.

Véron-Cetty, M. P. \& Véron, P., 1987. A Catalogue of Quasars and Active Nuclei, ESO Scientific Report, No. 5. 